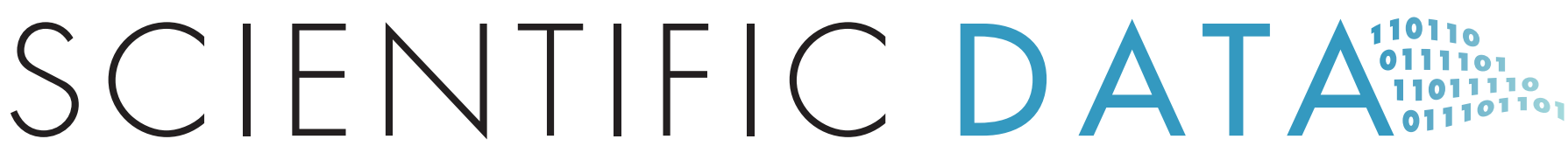

SUBJECT CATEGORIES

" Data mining

" Diagnosis

» Epidemiology

" Outcomes research :

Received: 16 June 2014 Accepted: 18 August 2014 Published: 16 September 2014

\section{Building the graph of medicine from millions of clinical narratives}

\author{
Samuel G. Finlayson, Paea LePendu \& Nigam H. Shah
}

Electronic health records (EHR) represent a rich and relatively untapped resource for characterizing the true nature of clinical practice and for quantifying the degree of inter-relatedness of medical entities such as drugs, diseases, procedures and devices. We provide a unique set of co-occurrence matrices, quantifying the pairwise mentions of 3 million terms mapped onto 1 million clinical concepts, calculated from the raw text of 20 million clinical notes spanning 19 years of data. Co-frequencies were computed by means of a parallelized annotation, hashing, and counting pipeline that was applied over clinical notes from Stanford Hospitals and Clinics. The co-occurrence matrix quantifies the relatedness among medical concepts which can serve as the basis for many statistical tests, and can be used to directly compute Bayesian conditional probabilities, association rules, as well as a range of test statistics such as relative risks and odds ratios. This dataset can be leveraged to quantitatively assess comorbidity, drug-drug, and drug-disease patterns for a range of clinical, epidemiological, and financial applications.

\begin{tabular}{|l|l|}
\hline Design Type(s) & clinical history design • data integration \\
\hline Measurement Type(s) & patient observation \\
\hline Technology Type(s) & medical examination \\
\hline Factor Type(s) & \\
\hline Sample Characteristic(s) & Homo sapiens • multi-cellular organism \\
\hline
\end{tabular}

Center for Biomedical Informatics Research, Stanford University, Stanford, California 94305, USA. Correspondence and requests for materials should be addressed to N.H.S. (email: nigam@stanford.edu) 


\section{Background \& Summary}

The widespread adoption of Electronic Health Records (EHRs) has enabled the longitudinal collection of patient health information at an unprecedented granularity and scale. As outlined by Jensen et al. ${ }^{1}$, efforts to mine this data show promise to impact nearly every aspect of healthcare. Nevertheless, these efforts are hindered, in part due to ethical and legal restrictions to data access. By tabulating clinical concept cooccurrences across millions of patient records and over a range of time windows (Figure 1), we provide rare quantitative glimpse into the degree of inter-relatedness of medical entities in large-scale clinical data.

Knowledge of such inter-relatedness can be readily leveraged for improving fundamental methods underlying a wide range of use cases, such as patient clustering ${ }^{2}$ and outcome prediction ${ }^{3}$, analysing comorbidity patterns ${ }^{4,5}$, and cohort querying for clinical trial design ${ }^{6}$. We provide here a brief overview of several areas that could employ the degree of inter-relatedness data we present.

Clinical concept co-occurrences can be used by clinical decision support (CDS) systems. The INTERNIST-1 system, its successor the Quick Medical Reference (QMR), and other diagnostic CDS utilities such as MYCIN and Pathfinder have been developed to aid physicians in diagnosing patients given their collections of symptoms ${ }^{7-11}$. Such systems generally depend on the painstaking manual development of knowledge bases to associate symptoms with diagnoses with varying degrees of confidence $^{12}$. The website for one new commercial CDS system, for example, advertises the application of more than 20,000 physician hours in the construction of such a graph ${ }^{13}$. Beyond the human burden for the initial population of such knowledge bases, manually curated datasets suffer from the rapid turnover in expert knowledge and the state of the literature, which leads to challenges in their upkeep or results in rapid out-dating, ${ }^{9,14}$. By leveraging co-occurrence data from real patient records, CDS systems could be populated with necessary conditional probabilities in a manner that is fast, scalable, easily updated, and by nature reflective of the patterns truly observed in clinical practice.

Clinical data allow probing population-wide comorbidity patterns over thousands of variables. As described by Valderas et al. . $^{15}$, although the notion of comorbidity is difficult to precisely define, the ability to quantitatively assess disease concurrence is of major interest within clinical, epidemiological, and financial health service contexts. Commonly, an individual's comorbidities are assessed by means of patient stratification or cumulative disease burden index, such as the Charlson Index ${ }^{16,17}$. Though specialized versions of such indices have been developed, such as transplantation ${ }^{18,19}$, the process of using custom studies to derive disease-specific comorbidity indices is un-scalable. In contrast, data-driven

a b

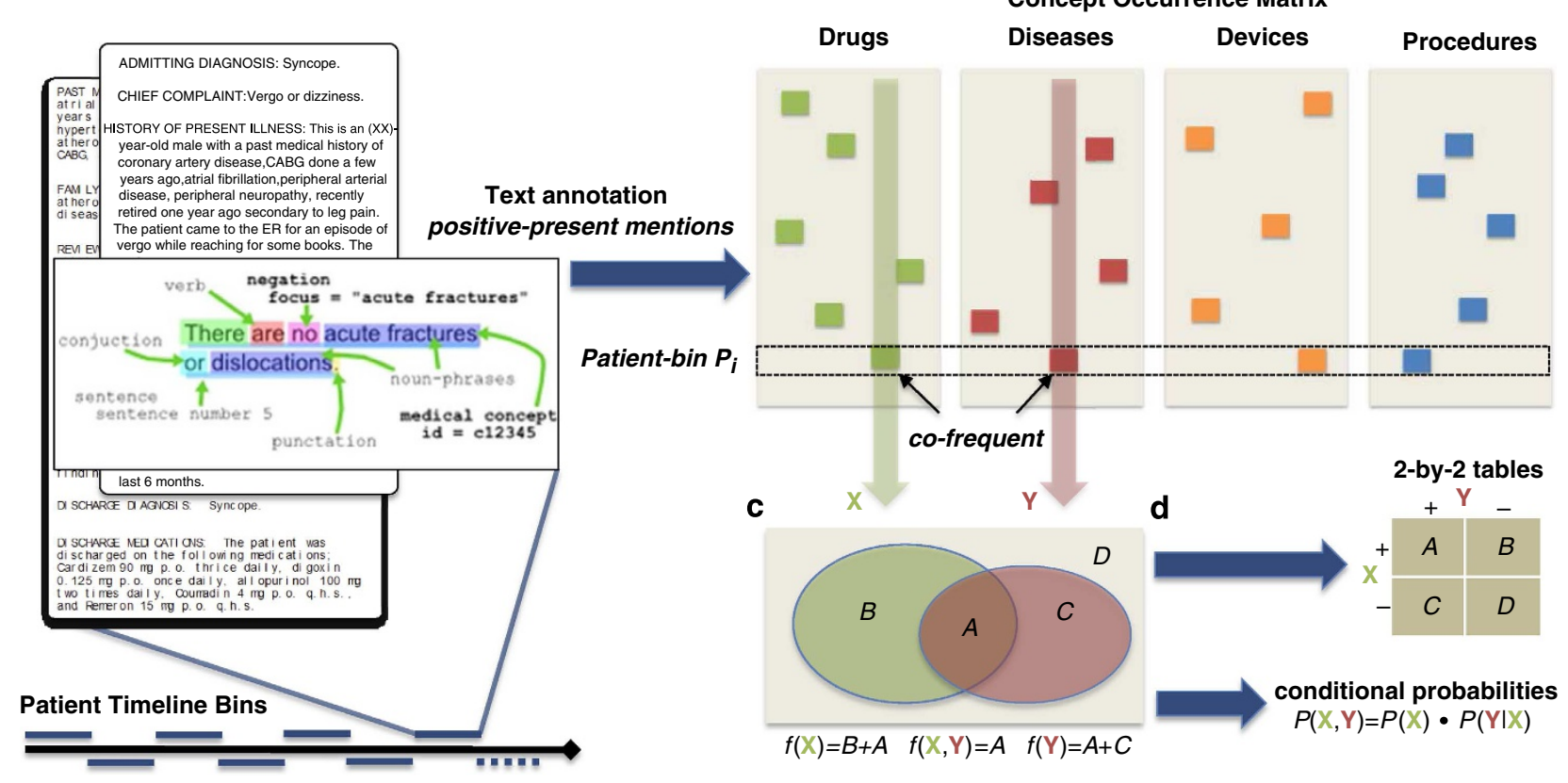

Figure 1. Workflow Architecture. The architecture of our workflow starts with (a) patient notes that are grouped together based on their nearness in time. Given the patient timeline bins, clinical terms are recognized from the notes and recorded into (b) the clinical concept occurrence matrix, which is scanned for (c) counting pairwise the frequency and co-frequency of concepts. This data can be used to calculate (d) contingency tables and Bayesian probability estimates. For example, the concept $X$ has a frequency of $f(X)$ and is pairwise co-frequent with concept $Y$ exactly $f(X, Y)$ times. 
approaches applied to comprehensive EHR datasets, such as that presented here, can learn comorbidity patterns across thousands of conditions ${ }^{4,5,20,21}$, thus complementing existing efforts.

Co-occurrence based analyses, which includes unstructured data sources in particular, can also enable new insights. For example, co-occurrence based disproportionality analysis across drug-disease pairings is employed for adverse drug event (ADE) detection in the field of computational pharmacovigilance. While many drug surveillance efforts focus on mining spontaneous reporting databases, given the underreporting in such databases ${ }^{22}$ and concern that coded data may miss $>90 \%$ of adverse drug events $^{23}$, recent efforts utilize unstructured EHR data for active pharmacovigilance ${ }^{24-26}$.

Examining disease co-occurrence patterns can enable the computational discovery of novel human health phenotypes ${ }^{4}$. Recent work has shown that many diseases classified historically as single entities are actually collections of many distinct phenotypes that may not match traditional disease boundaries ${ }^{20,27}$. Unsupervised learning methods applied to population-wide, comprehensive clinical datasets can redefine subtyping of existing diseases, and discover unrecognized phenotypes ${ }^{28-30}$. Our data can serve as the basis for building drug-drug, drug-disease, and disease-disease networks that can be utilized in such analyses.

The advent of larger datasets, including claims data, has given rise to high dimension propensity score methods that incorporate large numbers of variables for improved performance ${ }^{31}$. As the feature space for such analyses grows, so does the risk of latent instrumental or collider variables that could introduce Z- or M-biases ${ }^{32}$. Our co-occurrence counts can be used to infer latent dependencies between millions of clinical variables, which could spur the development of improved feature selection for high dimensional propensity scoring. Finally, inferred dependencies from our data could be used to assess the data assumptions of commonly used statistical methods, such as regression analysis, which commonly assumes feature independence.

\section{Methods}

\section{Data source}

We used clinical notes from the Stanford Translational Research Integrated Database Environment $(\text { STRIDE) })^{33}$. The STRIDE dataset contains 20 million unstructured clinical notes from 1.2 million patients, collected over a 19-year span. The notes comprise a combination of pathology, radiology, and transcription reports. We limited our calculation to notes from patients whose records contained a minimum of 10 notes spanning a time window of at least one year. The result was a raw set of 14 million text notes from 260 thousand patients.

\section{Note binning}

Each note contains terms that may be counted as co-occurring. However, we also employ a broader definition of concurrency by splicing together the contents of 'bins' of notes that are found to appear within a certain timeframe in a given patient's record. Figure 1a depicts how a single patients' set of notes can be spliced together over such bins.

Each note from STRIDE is time-stamped. For each patient, we used the time-stamps to hash notes into their temporal bins. Binning was accomplished via a simple hashing formula: For a patient $p$ and bin width $w$, we note $t_{0}$ (time of first record) and generated a random real number $0 \leq r \leq w$. We then defined a patient-specific hashing function operating on $w, r$, and a note's time $t$ (the note's delta from $t_{0}$ ):

$\mathrm{BIN}_{p}(w, r, t)=\left\lfloor\frac{t+r}{w}\right\rfloor$

For example, if $w=7$, then a note having a timestamp more than seven days later would necessarily be assigned a larger bin number. The random offset, $r$, was added to avoid potential global timestamp recording biases (e.g., a gone for the weekend effect).

For each patient's longitudinal health record, we consider 1, 7, 30, 90, 180, 365, and $\infty$-day bins. The bins are used to aggregate the frequency counts in two ways: (1) per bin, or (2) per patient. In the per-bin scenario, each relevant bin contributes a count; thus, one patient can contribute many counts. In the perpatient scenario, each patient contributes at most one count, no matter how many of his or her bins relate. Note that in the $\infty$-day bin these two scenarios are identical because each bin represents an entire patient record. Thus, each row in Figure $1 \mathrm{~b}$ either represents a bin or a patient depending on the aggregation scenario.

\section{Term extraction}

To extract the clinical concepts contained within each unstructured clinical note, we utilized the approach described previously by LePendu et al. ${ }^{24}$ See Figure 1a for an illustration of the concept recognition method. We first find mentions of terms from a dictionary compiled from 22 clinically relevant ontologies, such as SNOMED-CT and MedDRA. We apply a series of syntactic and semantic suppression rules to create a clean lexicon from these ontologies. We keep terms that are predominantly noun phrases based on an analysis of MEDLINE abstracts; we remove uninformative phrases based on term frequency analysis of over 50 million clinical documents from the Mayo clinic; and we suppress terms having fewer than four characters because these tend to be ambiguous abbreviations ${ }^{34-37}$. Finally, NegEx regular expressions are used to flag negative mentions (e.g., 'myocardial infarction was ruled out') and to determine if a term is mentioned in the history or family history section of the note ${ }^{38,39}$. We map the 
terms to concepts using the same 22 ontologies. During this term-concept mapping we identify ambiguous terms that belong to more than one semantic group (drug, disease, device, procedure) and suppress their least likely interpretation. For example 'clip' is more likely to be a device than a drug in clinical text, so we suppress the interpretation as 'Corticotropin-Like Intermediate Lobe Peptide. Further details on the text processing are found in LePendu et al. ${ }^{24}$

We filtered the list of terms by further applying a list of common stop words, as detailed in the Data Records section below. We additionally removed those terms that were shorter than four characters in length, as well as a manually curated list of terms that are homonymous for first or surnames. This final list of terms from each note was then indexed using Lucene ${ }^{40}$, and combined with the other notes in the same bin.

\section{Estimating the accuracy of the text processing pipeline}

We determine the accuracy of the event identification using a gold standard corpus from the 2008 i2b2 Obesity Challenge. This corpus has been manually annotated by two annotators for 16 conditions and was designed to evaluate the ability of NLP systems to identify a condition present for a patient given a textual note. We extended this corpus by manually annotating 9 additional outcomes. The list of 25 conditions evaluated is listed in supplemental information of LePendu et al. ${ }^{24}$

Using the set of terms corresponding to the definition of the concept of interest and the set of terms recognized by our annotation workflow in the i2b2 notes, we evaluate the sensitivity and specificity of identifying each of the conditions. Overall, our condition identification has $74 \%$ sensitivity and $96 \%$ specificity. Further details are found in LePendu et al. ${ }^{24}$

\section{Co-frequency counting}

A parallel processing approach was used to compute the co-frequencies on the terms within each bin (Figure 1b,c). This step was the most computationally expensive in the pipeline, being a $O\left(n * m^{2}\right)$ computation, where $n$ is the total number of bins and $m$ is the number of terms found within each bin. For example, for $w=7, n=5.5 \mathrm{M}$ and $m=200$, so the computation examines on the order of 220 billion pairs. A single pass over that many pairs takes approximately $9.5 \mathrm{~h}$ on a 20 -core machine with $117 \mathrm{~GB}$ of RAM. Table 1 lists summary statistics for each bin width.

Additionally, we provide a list of co-frequency counts computed over clinical concepts. For conceptlevel counting, we limited our data to terms that could be unambiguously mapped onto drugs or diseases. We then normalized all drugs to their ingredients using RxNorm, and normalized remaining terms to clinical concepts. We then counted the co-occurrences of the resulting concepts in the same manner as we counted the co-frequent terms. Finally, we removed rows from all files that contained counts of size 100 or fewer.

\section{Data Records}

The clinical text frequency dataset represents term and concept frequency counts over notes from 261,397 patients, computed over a range of bin widths (Data Citation 1). Summary data for the number and average size of the bins in each record are found in Table 1. Files are stored in tab-delimited format as outline below. To minimize file size, terms and concepts are stored using unique term and concept IDs; the keys are found in Data Record 3. We also provide Python scripts to assist in decoding these files (see Usage Notes). Filenames and specifications are found in the README.txt that accompanies the dataset. We uploaded the data to the Dryad Digital Repository (datadryad.org). Please see Data Citations for details.

\section{Data record 1-co-frequency counts}

The co-frequency counts are stored in 28 text files, each consisting of three tab-delimited columns. A row in each file represents a unique term-term or concept-concept pair and its counts, aggregated on

\begin{tabular}{|l|c|c|c|c|c|c|c|}
\hline & 1-day & 7-day & 30-day & 90-day & 180-day & 365 -day & $\infty$-day \\
\hline No. Bins & $7,334,261$ & $5,571,972$ & $3,969,069$ & $2,716,892$ & $2,014,460$ & $1,417,462$ & 261,397 \\
\hline No. Notes/Bin & 1.99 & 2.62 & 3.67 & 5.36 & 7.24 & 10.28 & 55.76 \\
\hline No. Terms/Bin & 169.48 & 200.70 & 246.12 & 305.91 & 363.36 & 447.20 & $1,417.36$ \\
\hline No. Concepts/Bin & 41.60 & 48.84 & 59.12 & 72.42 & 85.28 & 104.38 & 332.37 \\
\hline
\end{tabular}

Table 1. Summary statistics for each bin width. As bin width increases towards infinity, the total number of bins decreases, while the number of terms and concepts contained within each bin increases. The number of bins in the $\infty$-day window $(261,397)$ is equal to the total number of patients. 


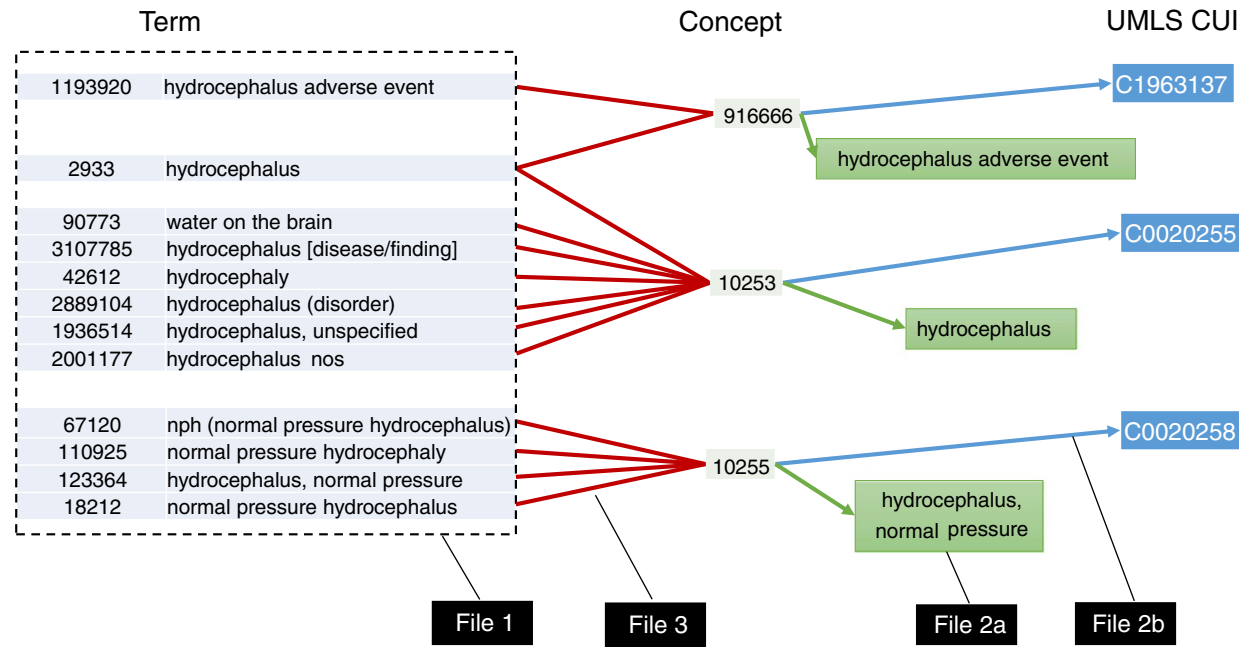

Figure 2. Mappings among terms and concepts. The figure explains the mappings that can be used to decode the frequency files stored in records 1 and 2. We use a subset of terms related to 'hydrocephalus' to demonstrate the mapping of terms (File 1) to concepts and UMLS CUIs. Terms map onto concepts in a many-to-many fashion (File 3). Concepts map onto CUIs in a one-to-one fashion (File 2 b) and have an associated string for human readability (File $2 \mathrm{a}$ ).

a per-bin or per-patient basis with a given bin width $w$. Terms and concepts are encoded using unique ID numbers, the maps for which are found in Data record 3.

\section{Data record 2-singleton frequency counts}

Singleton frequency counts are stored in 16 text files, each consisting of two tab-delimited columns. A row in each file represents a unique term or concept and its counts, aggregated on a per-bin or perpatient basis. Note that per-patient singleton counts are independent of bin width, and as such are only represented by a single file. The per-bin singleton counts, in contrast, are impacted by bin width and are as such represented by seven files, one per value of $w$. As with the co-frequency counts, terms and concepts are represented by integer term and concept IDs.

\section{Data record 3-term and concept ID mappings}

This record contains mappings that can be used to decode the frequency files stored in records 1 and 2 . The relationships captured in the different files are further explained in Figure 2. We also provide in this record the list of stop word strings that were repressed from the analysis.

File 1-term ID definitions. Each row pairs an integer term ID number with the string that it encodes in our dataset. For our purposes, a term is defined as a unique string.

File 2a-concept ID definitions: concept ID to string dictionary. Each row represents an integer concept ID and actual concept it represents in string form and the Unified Medical Language System (UMLS) Concept Unique Identifier (CUI) for that concept.

File $2 \mathrm{~b}$-concept ID definitions: concept ID to CUI dictionary. Each row represents an integer concept ID and the UMLS CUI for that concept.

File 3-term to concept dictionary. A many-to-many mapping of term IDs to concept IDs. Any terms whose mapping onto concept is not listed was suppressed from the concept co-frequency counts.

File 4-stop words. The list of words treated as stop words in the analysis. The stop words list also contains the list of terms that are homonymous for first or surnames, which are excluded from the counting.

\section{Technical Validation}

The annotation pipeline has been previously validated, as described in LePendu et al. ${ }^{24}$ We used simulation to assess the accuracy of our co-frequency counting methodology. We wrote a program that generates known term bins along with their correct co-occurrence counts. We simulated over 1,000 random 'notes' for 100 randomly simulated patients and computed the bins, and calculated the co-frequency counts at the bin and patient level using two different methods. We first wrote a 
single-threaded python script to take the simulated notes and count the co-frequencies basically duplicating the functionality of the system, but without using any parallelization. In parallel we ran cofrequency counter using Spark that used a different approach to parallelization than the one we used in the manuscript. The co-frequency counts from the two methods agreed perfectly with the parallelized method we implemented. Because we are calculating co-frequencies at a large scale using a distributed process, we focused on validating the transformation of the annotated text into bins, and into co-frequencies using a simulated dataset (where the answer is known) and two different approaches for comparison.

\section{Usage Notes}

Mapping terms to concepts and strings to CUIS

For convenience, we provide Python scripts, 'decode_cofreqs.py' and 'decode_singlets.py' that can be used to transform the term and concept IDs from a co- or singleton-frequency file into their corresponding strings and CUIs. These scripts can be adjusted or incorporated into an analysis pipeline, and follow the form:

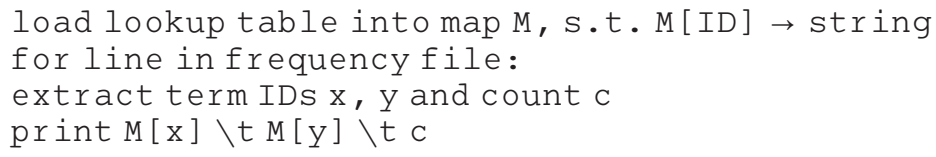

These scripts require only a Data Record 1 frequency file and a Data Record 2 dictionary file to complete the mapping. Example command line usage for the scripts is found within their header comments.

\section{Computing contingency tables}

Co-occurrence data can be leveraged to build contingency tables (Figures 1c,d and 3). Contingency tables can be directly utilized in statistical tests, such as odds ratio, relative risk, the G-test, enrichment analysis and the chi-squared test ${ }^{41}$. In constructing contingency tables, our co-frequency counts can be utilized for hypothesis testing across many variables that are typically missing from large observational datasets, which can in turn motivate traditional research studies. Obviously multiple hypothesis testing becomes an issue that needs to be addressed ${ }^{42}$.

For example, in Figure 3, for two terms or concepts $\mathrm{X}$ and $\mathrm{Y}$, their co-frequency is denoted $f(\mathrm{X}, \mathrm{Y})$ and would go into cell ' $A$ ' of the 2-by-2 contingency table. Visualized as a Venn diagram, cell ' $B$ ' is the frequency of $\mathrm{X}$ minus the intersection: $f(\mathrm{X})-f(\mathrm{X}, \mathrm{Y})$. Cell ' $D$ ' of the contingency table is calculated by subtracting $A, B$, and $C$ from the universe $N$, which is the number of bins for the given width (row 1 of Table 1). Specifically, $\mathrm{D}=N-(A+B+C)$ or $\mathrm{D}=N-[f(\mathrm{X})+f(\mathrm{Y})]+f(\mathrm{X}, \mathrm{Y})$. If computing over per-patient counts, use the $\infty$-day bin for $N$, which is the number of patients $(N=261,397)$. The resulting table can then be plugged into any standard statistical package.

\section{Estimating bayesian probabilities}

The data we present can also be leveraged in Bayesian networks ${ }^{43}$ (Figure 1d), which have been described in the literature as integral components of clinical decision support systems $s^{7,8,44-46}$. The basic Bayesian probabilities that form the basis for such methods can be inferred directly from our data. For example, given two clinical terms $\mathrm{X}, \mathrm{Y}$ and a total number $\mathrm{N}$ of bins or patients (see section on contingency tables), one might compute:

$P(X)=\frac{f(X)}{N} P(X, Y)=\frac{f(X, Y)}{N} P(Y \mid X)=\frac{P(X, Y)}{P(X)}$

These probabilities can then be used to make additional estimates and inferences. For example, the lift of the association rule $\mathrm{X} \rightarrow \mathrm{Y}$, which functions as a measure of statistical dependence between $\mathrm{X}$ and $\mathrm{Y}$,

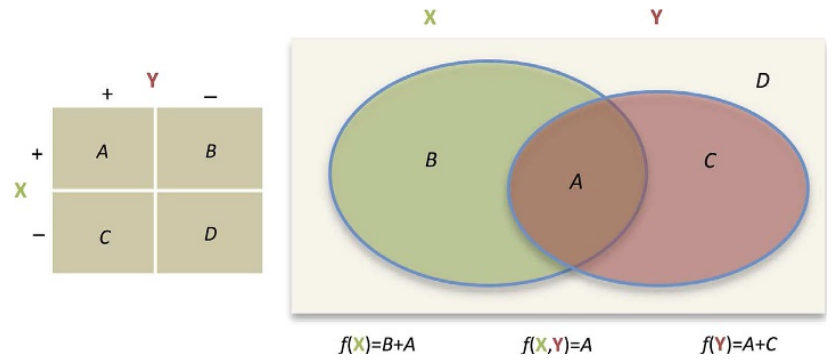

Figure 3. Filling out the 2-by-2 contingency table. 
can be computed as follows:

$\operatorname{Lift}(X \rightarrow Y)=\frac{P(Y \mid X)}{P(Y)}$

The lift could then be utilized, for example, as a filter to suppress spurious findings in a disproportionality analysis or to assist in identifying $\mathrm{M}$ - or Z- bias in propensity score or regression models ${ }^{31,32}$.

Our co-frequency counts can be used to construct networks of concept-concept associations in healthcare data, which can be employed to detect and eliminate variables that may introduce error in healthcare propensity models.

\section{Defining groups of related features}

Statistical models to learn clinical phenotypes must incorporate a large number of variables, many of which are highly collinear ${ }^{32}$. Similar to using lift, one might leverage co-frequency counts as a component of a group LASSO penalty ${ }^{47}$ in building better phenotyping models. Regularized regression methods such as the LASSO can be employed to reduce the feature space and enhancements such as the group LASSO can enforce sparsity both within and between defined sets of variables. Co-frequency counts can be used to define the groups of highly correlated variables over which the group LASSO penalty is applied. Similar approaches can be employed in the construction of statistical models with applications other than phenotyping, such as outcome prediction or risk assessment-in fact, any model that is trained over large numbers of clinical variables and is subject to assumptions of feature independence may benefit from a quantitative characterization of feature co-linearity from our data.

\section{Improved enrichment analysis}

Enrichment Analysis, which determines whether the Gene Ontology (GO) terms about specific biological processes, molecular functions, or cellular components are over- or under-represented in the annotations of the genes considered differentially expressed ${ }^{48}$, is commonly used to gain insight into the biological significance of alterations in gene expression levels ${ }^{49}$, as well as to profile other genome scale data such as tissue-microarrays ${ }^{50,51}$.

Recently, enrichment analysis has been performed using disease ontologies, such as SNOMED-CT, as well as being applied to other datasets of interest, such as patient cohorts. For example, by annotating protein mutations with disease terms, Mort et al. ${ }^{52}$ identified a class of diseases-blood coagulation disorders - that are associated with a significant depletion in substitutions at O-linked glycosylation sites. In another example, enrichment analysis was used detect specific co-morbidities that have an increased incidence in rheumatoid arthritis patients, a topic of recent discussion in the literature and considered essential to provide high quality care ${ }^{53-55}$.

Among the different limitations of enrichment analysis, a crucial one is the handling of dependence between annotations, e.g., annotation of an EHR with hypertension and lisinopril or diabetes are not independent because lisinopril is a drug that treats hypertension and diabetes is a common co-morbidity. The dependencies among annotations with terms from different branches of the GO are well $\mathrm{known}^{49,56,57}$. For example, genes annotated with the molecular function of electron transport are also annotated with the biological process of cellular respiration because electron transport occurs during cellular respiration. However, the dependencies between occurrences of medical concepts in patient records are not well quantified.

Methods to account for dependency among terms typically rely on co-occurrence frequency of terms in an annotation corpus. The intuition is that we can increase the 'expectedness' of a term based on the co-occurrence of that term with other terms in a large reference annotation set. Using the co-occurrence of the two terms, we can quantify the increased expectation and adjust the $P$-values accordingly. The dataset we provide allows researchers to account for annotation dependency via adjusting the enrichment calculation, by accounting for the overlap of two sets X (items annotated with $x$ ) and Y (items annotated with $y$ ) if the intersection A (items annotated with $x$ and $y$ ) is known, as described by Grossman et al. ${ }^{58}$

In summary, electronic health records represent a rich and untapped resource in analysing the true nature of clinical practice. EHR-powered research is limited by privacy and security concerns that limit data sharing. While these limitations make sharing of our raw data impossible, we provide co-occurrence counts derived from processed clinical notes. These co-occurrence matrices can be mined directly for cooccurrence patterns of interest, can be leveraged to improve the performance and generalizability of statistical models with a large number of features, and can enable improved enrichment analyses of patient cohorts.

\section{References}

1. Jensen, P. B., Jensen, L. J. \& Brunak, S. Mining electronic health records: towards better research applications and clinical care. Nat. Rev. Genet. 13, 395-405 (2012).

2. Cole, T. S. et al. Profiling risk factors for chronic uveitis in juvenile idiopathic arthritis: a new model for EHR-based research. Pediatr. Rheumatol. Online J. 11, 45 (2013).

3. Saria, S. et al. Integration of early physiological responses predicts later illness severity in preterm infants. Sci. Transl. Med. 2, $48 \mathrm{ra} 65$ (2010). 
4. Blair, D. R. et al. A nondegenerate code of deleterious variants in mendelian Loci contributes to complex disease risk. Cell 155, 70-80 (2013).

5. Rzhetsky, A. et al. Probing genetic overlap among complex human phenotypes. Proc. Natl Acad. Sci. USA 104, 11694-11699 (2007).

6. Pathak, J., Kho, A. N. \& Denny, J. C. Electronic health records-driven phenotyping: challenges, recent advances, and perspectives. J. Am. Med. Inform. Assoc. 20, e206-e211 (2013).

7. Heckerman, D. E., Horvitz, E. \& Nathwani, B. N. Toward normative expert systems: Part I. The Pathfinder Project. Methods Inf. Med. 31, 90-105 (1992).

8. Heckerman, D. E. \& Nathwani, B. N. Toward normative expert systems: Part II. Probability-based representations for efficient knowledge acquisition and inference. Methods Inf. Med. 31, 106-116 (1992).

9. Miller, R. A history of the INTERNIST-1 and Quick Medical Reference (QMR) computer-assisted diagnosis projects, with lessons learned. Yearb. Med. Inform. 121-136 (2010).

10. Miller, R. A. et al. The INTERNIST-1/quick medical REFERENCE project-Status report. West. J. Med. 145, 816 (1986).

11. Shortliffe, E. H. MYCIN: Computer-based Medical Consultations (Elsevier, 1976).

12. Shwe, M. A. et al. Probabilistic diagnosis using a reformulation of the INTERNIST-1/QMR knowledge base. Meth. Inform. Med. 30, 241-255 (1991).

13. Lumiata, Lumiata Raises \$4 Million in Series A Financing from Khosla Ventures http://lumiata.com/press-release/ (2013).

14. Waitman, L. R. \& Miller, R. A. Pragmatics of implementing guidelines on the front lines. J. Am. Med. Inform. Assoc. 11, 436-438 (2004)

15. Valderas, J. M. et al. Defining comorbidity: implications for understanding health and health services. Ann. Fam. Med. 7, 357-363 (2009).

16. Charlson, M. E. et al. A new method of classifying prognostic comorbidity in longitudinal studies: development and validation. J. Chronic Dis. 40, 373-383 (1987).

17. de Groot, V. et al. How to measure comorbidity. a critical review of available methods. J. Clin. Epidemiol. 56, 221-229 (2003).

18. Sorror, M. L. et al. Hematopoietic cell transplantation (HCT)-specific comorbidity index: a new tool for risk assessment before allogeneic HCT. Blood 106, 2912-2919 (2005).

19. Volk, M. L. et al. Modified Charlson comorbidity index for predicting survival after liver transplantation. Liver Transpl. 13, 1515-1520 (2007).

20. Wenzel, S. E. Asthma phenotypes: the evolution from clinical to molecular approaches. Nat. Med. 18, 716-725 (2012).

21. Holmes, A. B. et al. Discovering disease associations by integrating electronic clinical data and medical literature. PLoS ONE 6, e21132 (2011).

22. Lopez-Gonzalez, E., Herdeiro, M. T. \& Figueiras, A. Determinants of under-reporting of adverse drug reactions. Drug Safety 32, 19-31 (2009).

23. Classen, D. C. et al. 'Global trigger tool' shows that adverse events in hospitals may be ten times greater than previously measured. Health Affairs 30, 581-589 (2011).

24. LePendu, P. et al. Annotation analysis for testing drug safety signals using unstructured clinical notes. J. Biomed. Semantics 3 (Suppl 1): S5 (2012).

25. LePendu, P. et al. Analyzing patterns of drug use in clinical notes for patient safety. AMIA Summits Transl. Sci. Proc. 2012, 63 (2012).

26. Stang, P. E. et al. Advancing the science for active surveillance: rationale and design for the Observational Medical Outcomes Partnership. Ann. Intern. Med. 153, 600-606 (2010).

27. De Keulenaer, G. W. \& Brutsaert, D. L. The heart failure spectrum time for a phenotype-oriented approach. Circulation 119, 3044-3046 (2009).

28. Kohane, I. S. The twin questions of personalized medicine: who are you and whom do you most resemble? Genome Med. 1, 4 (2009).

29. Lasko, T. A., Denny, J. C. \& Levy, M. A. Computational phenotype discovery using unsupervised feature learning over noisy, sparse, and irregular clinical data. PLoS ONE 8, e66341 (2013).

30. National Research Council Committee on, A.F.f.D.a.N.T.o.D. The National Academies Collection: Reports funded by National Institutes of Health, in Toward Precision Medicine: Building a Knowledge Network for Biomedical Research and a New Taxonomy of Disease (National Academies Press, 2011).

31. Schneeweiss, S. et al. High-dimensional propensity score adjustment in studies of treatment effects using health care claims data. Epidemiology 20, 512-522 (2009).

32. Brookhart, M. A. et al. Confounding control in healthcare database research: challenges and potential approaches. Med. Care 48 (6 Suppl): S114-S120 (2010).

33. Lowe, H. J. et al. STRIDE-An integrated standards-based translational research informatics platform. in AMIA Annu. Symp. Proc. 2009, 391-395 (2009).

34. Bodenreider, O. \& McCray, A. T. Exploring semantic groups through visual approaches. J. Biomed. Inform. 36, 414-432 (2003).

35. Parai, G. K. et al. The Lexicon Builder Web Service: building custom lexicons from two hundred biomedical ontologies. in AMIA Annu. Symp. Proc. 2010, 587-591 (2010).

36. Wu, S. T. et al. Unified Medical Language System term occurrences in clinical notes: a large-scale corpus analysis. J. Am. Med. Inform. Assoc. 19, e149-e156 (2012).

37. Xu, R., Musen, M. A. \& Shah, N. H. A comprehensive analysis of five million UMLS metathesaurus terms using eighteen million MEDLINE citations. in AMIA Annu. Symp. Proc. 2010, 907-911 (2010).

38. Chapman, W.W. et al. A simple algorithm for identifying negated findings and diseases in discharge summaries. J. Biomed. Inform. 4, 301-310 (2001).

39. Chapman, W. W., Chu, D. \& Dowling, J. N. BioNLP '07 Proceedings of the Workshop on BioNLP 2007: Biological, Translational, and Clinical Language Processing, 81-88 (Association for Computational Linguistics Stroudsburg, PA, USA, 2007).

40. Lucene, A. A high-performance, full-featured text search engine library. http://lucene.apache.org (2005).

41. Sauerbrei, W. \& Blettner, M. Interpreting results in $2 \times 2$ tables: part 9 of a series on evaluation of scientific publications. Dtsch. Arztebl. Int. 106, 795-800 (2009).

42. Sainani, K. L. The problem of multiple testing. PM\&R 1, 1098-1103 (2009).

43. Pearl, J. Probabilistic Reasoning in Intelligent Systems: Networks of Plausible Inference (Morgan Kaufmann, 1988).

44. Chen, J. \& Altman, R. B. Automated Physician Order Recommendations and Outcome Predictions by Data-Mining Electronic Medical Records. AMIA Summit Transl. Bioinform. 206-210 (2014).

45. Klann, J., Schadow, G. \& Downs, S. M. A method to compute treatment suggestions from local order entry data. in AMIA Annu. Symp. Proc. 2010, 387-391 (2010).

46. Klann, J., Schadow, G. \& McCoy, J. A recommendation algorithm for automating corollary order generation. in AMIA Annu. Symp. Proc. 2009, 333-337 (2009). 
47. Simon, N. et al. A sparse-group lasso. J. Comp. Graph. Stat. 22, 231-245 (2013).

48. Zeeberg, B. R. et al. GoMiner: a resource for biological interpretation of genomic and proteomic data. Genome Biol. 4, R28 (2003).

49. Rhee, S. Y. et al. Use and misuse of the gene ontology annotations. Nat. Rev. Genet. 9, 509-515 (2008).

50. Tirrell, R. et al. An ontology-neutral framework for enrichment analysis. AMIA Annu. Symp. Proc. 2010, 797-801 (2010).

51. Shah, N. H. et al. Annotation and query of tissue microarray data using the NCI Thesaurus. BMC Bioinformatics 8, 296 (2007).

52. Mort, M. et al. In silico functional profiling of human disease-associated and polymorphic amino acid substitutions. Human Mutation 31, 335-346 (2010).

53. Michaud, K. \& Wolfe, F. The association of rheumatoid arthritis and its treatment with sinus disease. J. Rheumatol. 33, $2412-2415$ (2006).

54. Turesson, C., Jacobsson, L. T. \& Matteson, E. L. Cardiovascular co-morbidity in rheumatic diseases. Vasc. Health Risk Manag. 4, 605-614 (2008).

55. John, H. et al. Cardiovascular co-morbidity in early rheumatoid arthritis. Best Pract. Res. Clin. Rheumatol. 23, 71-82 (2009).

56. Myhre, S. et al. Additional gene ontology structure for improved biological reasoning. Bioinformatics 22, 2020-2027 (2006).

57. Huang da, W., Sherman, B. T. \& Lempicki, R. A. Bioinformatics enrichment tools: paths toward the comprehensive functional analysis of large gene lists. Nucleic Acids Res. 37, 1-13 (2009).

58. Grossmann, S. et al. Improved detection of overrepresentation of Gene-Ontology annotations with parent child analysis. Bioinformatics 23, 3024-3031 (2007).

\section{Data Citation}

1. Finlayson, S. G., LePendu, P. \& Shah, N. H. Dryad http://dx.doi.org/10.5061/dryad.jp917 (2014).

\section{Acknowledgements}

The authors acknowledge support from the National Institutes of Health grants U54-HG004028 and R01-LM011369.

\section{Author Contributions}

S.G.F. wrote all of the programs for computing the co-frequencies under supervision by P.L. P.L. and N.H.S. conceived the work. S.G.F., P.L., and N.H.S. equally contributed to the manuscript writing.

\section{Additional information}

Competing financial interests: The authors declare no competing financial interests.

How to cite this article: Finlayson, S. G. et al. Building the graph of medicine from millions of clinical narratives. Sci. Data 1:140032 doi: 10.1038/sdata.2014.32 (2014).

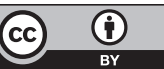

This work is licensed under a Creative Commons Attribution 4.0 International License. The images or other third party material in this article are included in the article's Creative Commons license, unless indicated otherwise in the credit line; if the material is not included under the Creative Commons license, users will need to obtain permission from the license holder to reproduce the material. To view a copy of this license, visit http://creativecommons.org/licenses/by/4.0

Metadata associated with this Data Descriptor is available at http://www.nature.com/sdata/ and is released under the CCO waiver to maximize reuse. 